\title{
Using HOESY NMR spectroscopy to characterise pre-nucleation aggregates
}

Raitis Bobrovs*a, Andrievs Auseklis Auzinsa, Laura Drunkaa, Rimants Metlansa, Ruslans Muhamadejevs ${ }^{\mathrm{a}}$ and Kristaps Jaudzems ${ }^{\mathrm{a}, \mathrm{b}}$

a Latvian Institute of Organic Synthesis, Aizkraukles 21, LV-1006, Riga, Latvia

b Faculty of Chemistry, University of Latvia, Jelgavas 1, LV-1004 Riga, Latvia

*Email: raitis.bobrovs@osi./v

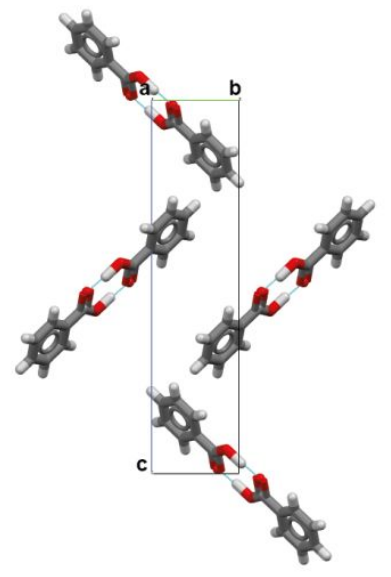

BA

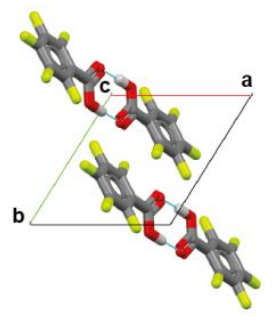

pFBA

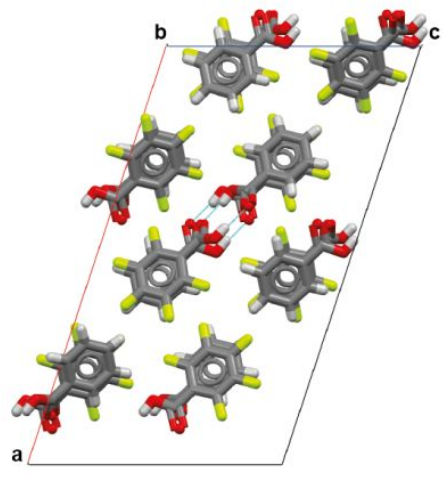

pFBA-BA co-crystal

Fig. SI1. Crystal packing motifs of benzoic acid (BA), pentafluorobenzoic acid ( $p F B A$ ) and their cocrystal. Turquoise dashed lines represents hydrogen bonds.

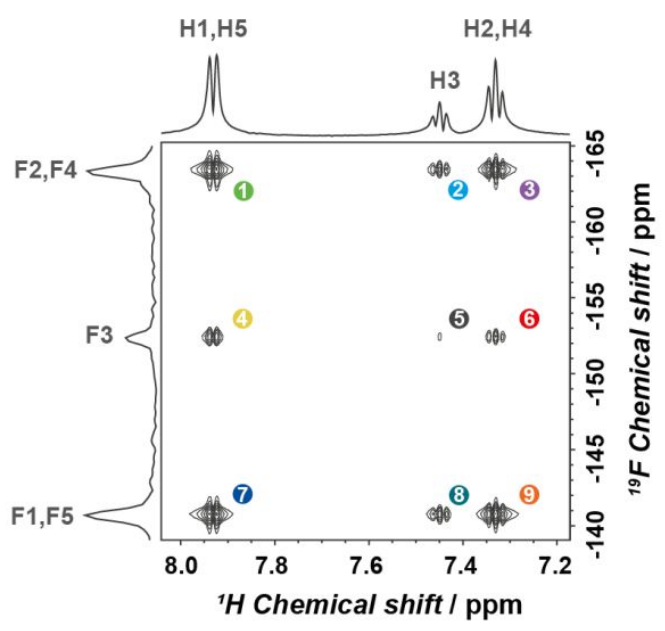

A

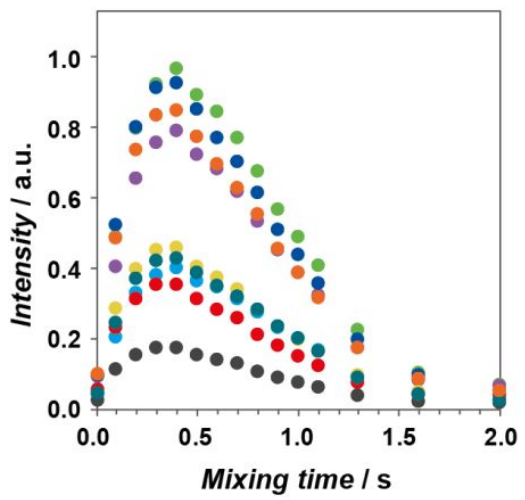

B

Fig. SI2. A. ${ }^{19} \mathrm{~F}^{-1} \mathrm{H}$ HOESY spectrum of $2 \mathrm{M} B A$ pFBA $1: 1$ solution in methanol-D ${ }_{4}$ B. ${ }^{19} \mathrm{~F}^{-1} \mathrm{H} H O E$ signal intensity as a function of the HOESY experiment mixing time. The HOE signal colour coding is indicated in subfigure $A$. 

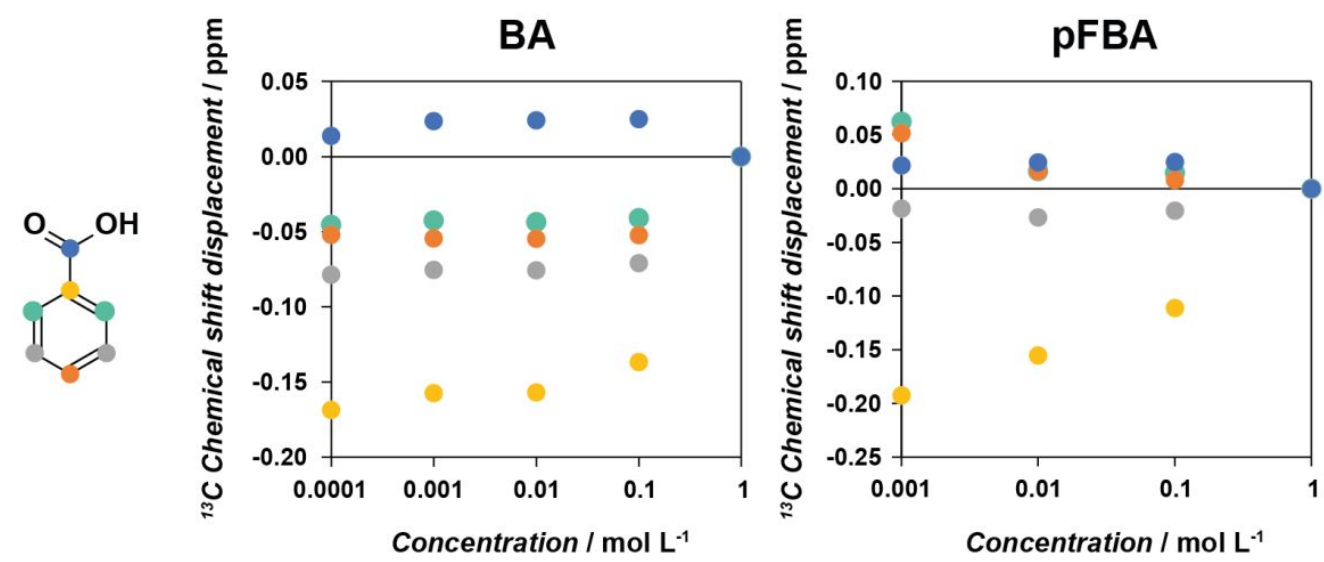

Fig. SI3. $B A$ and $p F B A{ }^{13} C$ chemical shift displacement $(\triangle \delta$ with respect to the most concentrated solution) as a function of methanol- $D_{4}$ solution concentration.

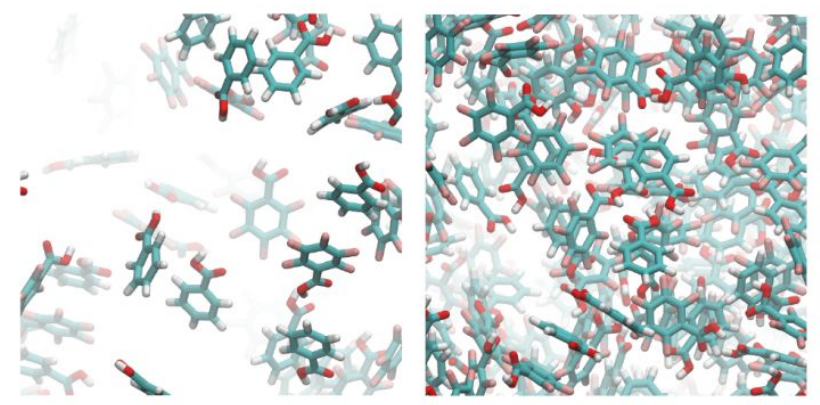

A

$\mathbf{B}$

Fig. SI4. MD simulation snapshots of (A) 0.5 and (B) 2 M 1:1 BA pFBA solution in methanol. Methanol molecules are omitted for clarity.

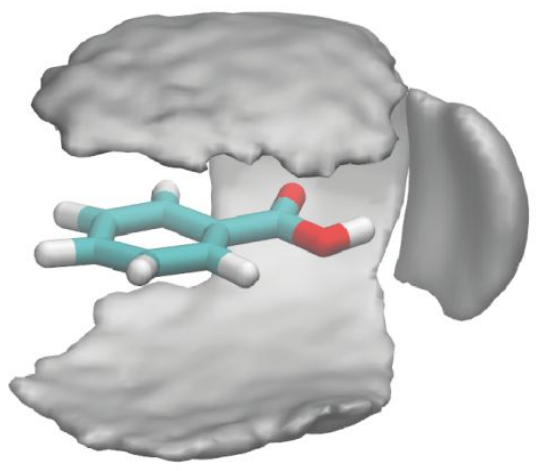

Fig. SI5. The spatial distribution function of pFBA molecules around BA molecule in $2.0 \mathrm{M}$ methanol solution (distance cut-off 10 A). 
Table SI1. Fraction (\%) of BA and pFBA molecules involved in $\Pi-\pi$ stacked and hydrogen bonded aggregate formation, mean duration of the interaction and distribution into specific aggregate subtype.

\begin{tabular}{lllllll} 
c / M & $\mathbf{0 . 1}$ & $\mathbf{0 . 3}$ & $\mathbf{0 . 5}$ & $\mathbf{1 . 0}$ & $\mathbf{2 . 0}$ & $\mathbf{2 . 5}$ \\
\hline monomers & 89.7 & 71.9 & 56.1 & 23.4 & 7.6 & 3.6 \\
\hline $\begin{array}{l}\text { H-bonding (symmetric*) } \\
\text { H-bonding mean duration, }\end{array}$ & $1.3(10.1)$ & $3.8(11.6)$ & $7.7(10.5)$ & $15.1(12.7)$ & $32.8(16.2)$ & $44.0(14.6)$ \\
ps & 68.0 & 71.1 & 78.0 & 93.0 & 108.0 & 115.9 \\
\hline n-n stacking & 4.1 & 12.1 & 20.0 & 36.0 & 63.8 & 72.9 \\
$\begin{array}{l}\text { T-shaped } \\
\text { face-to-face }\end{array}$ & 16.1 & 16.5 & 16.9 & 16.9 & 18.2 & 20.1 \\
$\begin{array}{l}\text { offset } \\
\text { intermediate }\end{array}$ & 4.5 & 4.4 & 4.5 & 4.4 & 4.4 & 3.9 \\
$\begin{array}{l}\text { n-ח stacking mean } \\
\text { duration, ps }\end{array}$ & 18.1 & 60.8 & 60.4 & 60.8 & 60.0 & 57.9 \\
\hline
\end{tabular}

* Fraction of all hydrogen bonded solute molecules.

** One solute molecule can be involved in both hydrogen bonding and $\Pi-n$ stacking (with more than one molecule) simultaneously, therefore total fraction of self-associated molecules can exceed $100 \%$. At the same time, two molecules can be close enough to each other not to be identified as monomers, while not satisfying conditions of a specific aggregate.

Table SI2. The fraction (\%) of BA and pFBA molecules involved in $\Pi-\pi$ stacked and hydrogen bonded aggregate formation, life-time of these aggregates and distribution into specific aggregate subtype in various solvents.

\begin{tabular}{|c|c|c|c|c|c|c|c|c|c|}
\hline Solvent & 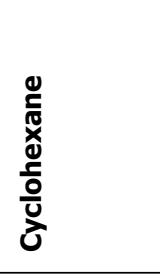 & 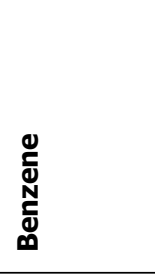 & 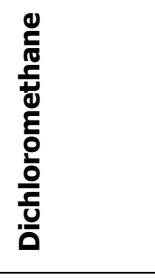 & 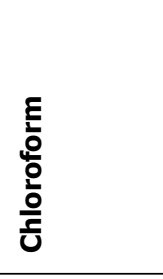 & & 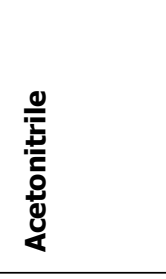 & 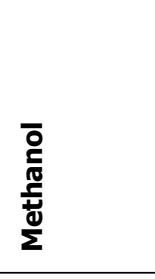 & 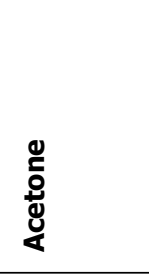 & 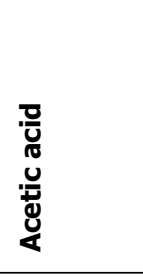 \\
\hline monomers & 0.8 & 15.2 & 6.5 & 6.2 & 54.4 & 30.3 & 56.1 & 54.1 & 60.3 \\
\hline H-bonding (symmetric) & $67.1(38.8)$ & $53.1(34.2)$ & $57.2(45.3)$ & $60.6(40.0)$ & $13.7(24.8)$ & $44.9(30.4)$ & $7.7(10.5)$ & $8.1(19.0)$ & $5.9(10.2)$ \\
\hline $\begin{array}{l}\mathrm{H} \text {-bonding mean } \\
\text { duration, ps }\end{array}$ & 353.9 & 317.2 & 176.9 & 256.7 & 211.0 & 149.5 & 78.0 & 140.7 & 120.4 \\
\hline ח-п stacking & 45.6 & 13.2 & 19.3 & 19.7 & 14.6 & 25.5 & 20.0 & 15.6 & 17.8 \\
\hline T-shaped & 16.6 & 22.8 & 15.7 & 16.1 & 20.8 & 23.3 & 16.9 & 19.3 & 19.0 \\
\hline face-to-face & 3.1 & 3.2 & 4.0 & 4.1 & 3.4 & 3.5 & 4.5 & 2.9 & 4.3 \\
\hline offset & 62.7 & 54.8 & 62.2 & 61.7 & 57.4 & 53.1 & 60.4 & 57.1 & 58.3 \\
\hline intermediate & 17.6 & 19.1 & 18.0 & 18.1 & 18.3 & 20.1 & 18.1 & 20.7 & 18.3 \\
\hline $\begin{array}{l}\text { n- } \Pi \text { stacking mean } \\
\text { duration, ps }\end{array}$ & 67.5 & 47.3 & 38.9 & 43.6 & 46.8 & 39.1 & 41.8 & 38.4 & 65.3 \\
\hline
\end{tabular}

* One solute molecule can be involved in both hydrogen bonding and $п-n$ stacking (with more than one molecule) simultaneously, therefore total fraction of self-associated molecules can exceed $100 \%$. At the same time, two molecules can be close enough to each other not to be identified as monomers, while not satisfying conditions of a specific aggregate. 

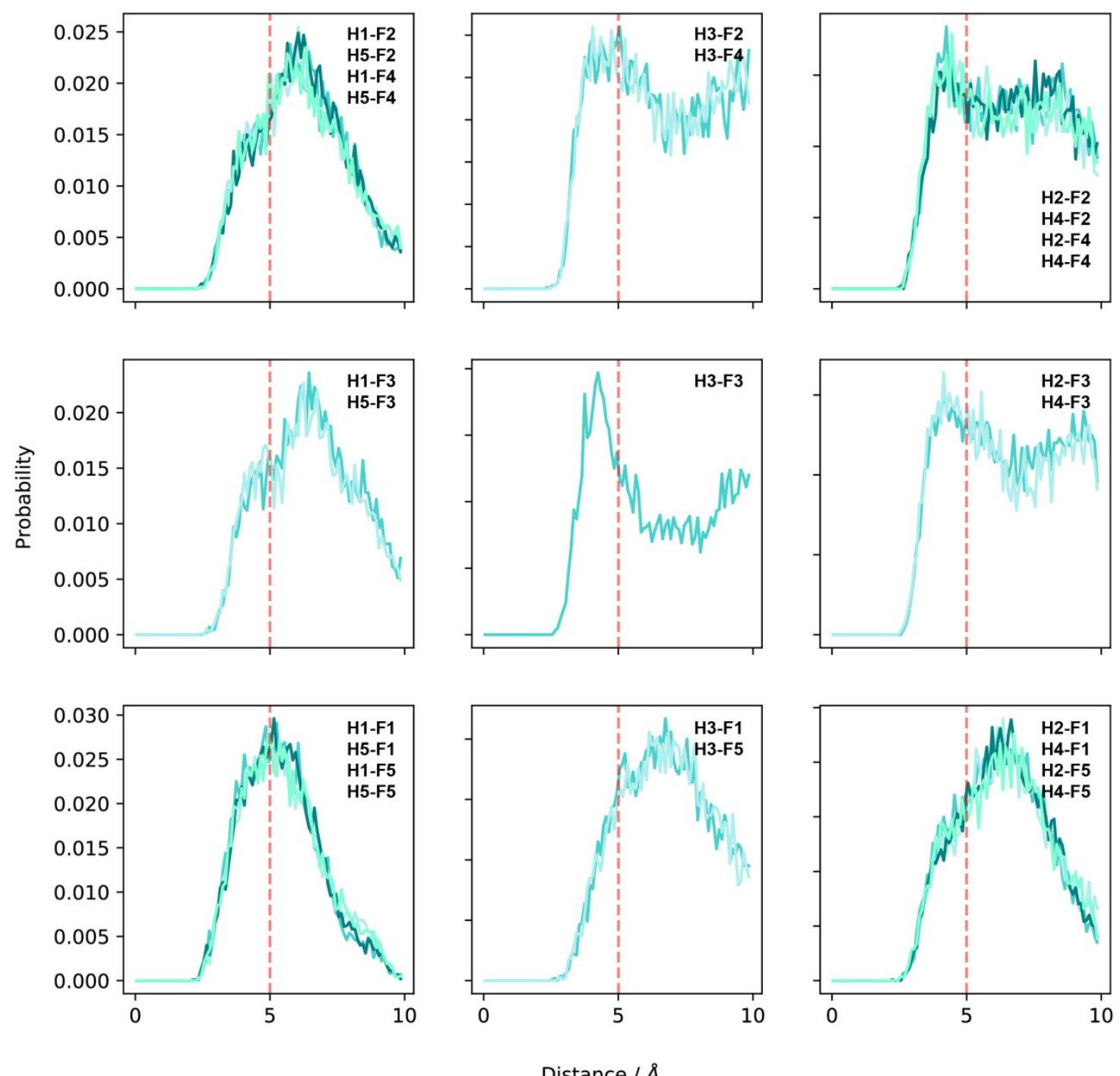

Distance / $\AA$

Fig. SI6. Radial pair distribution function of equivalent aromatic $H$ and $F$ pairs in hydrogen bonded $B A-P F B A$ dimers (both symmetric and asymmetric, as observed in MD simulations). Red dashed line represents HOE distance cut-off $(5 \AA)$. 

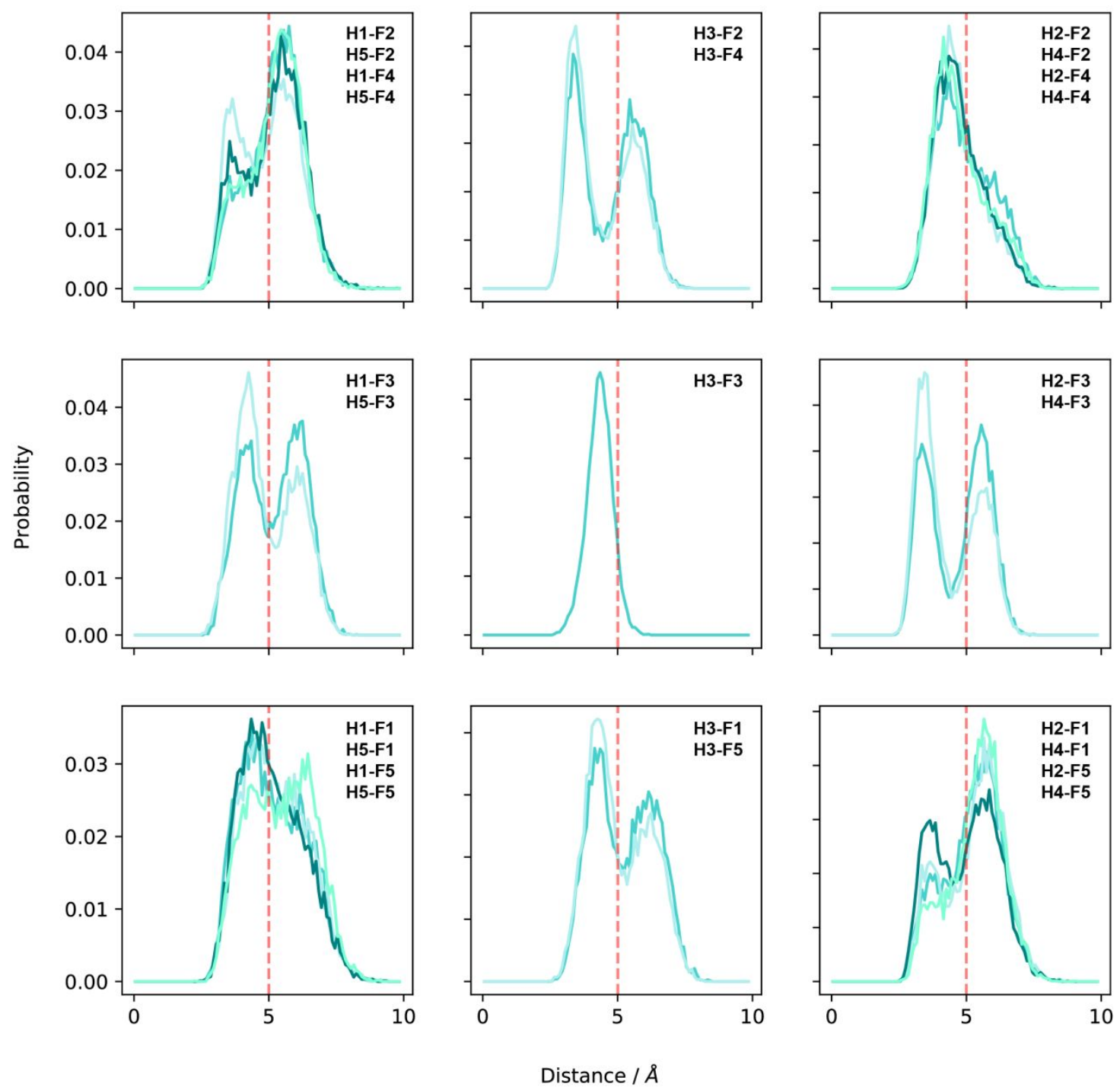

Fig. SI7. Radial pair distribution function of equivalent aromatic $H$ and $F$ pairs in $\pi-\pi$ stacked BAPFBA dimer. Red dashed line represents HOE distance cut-off (5 A). 


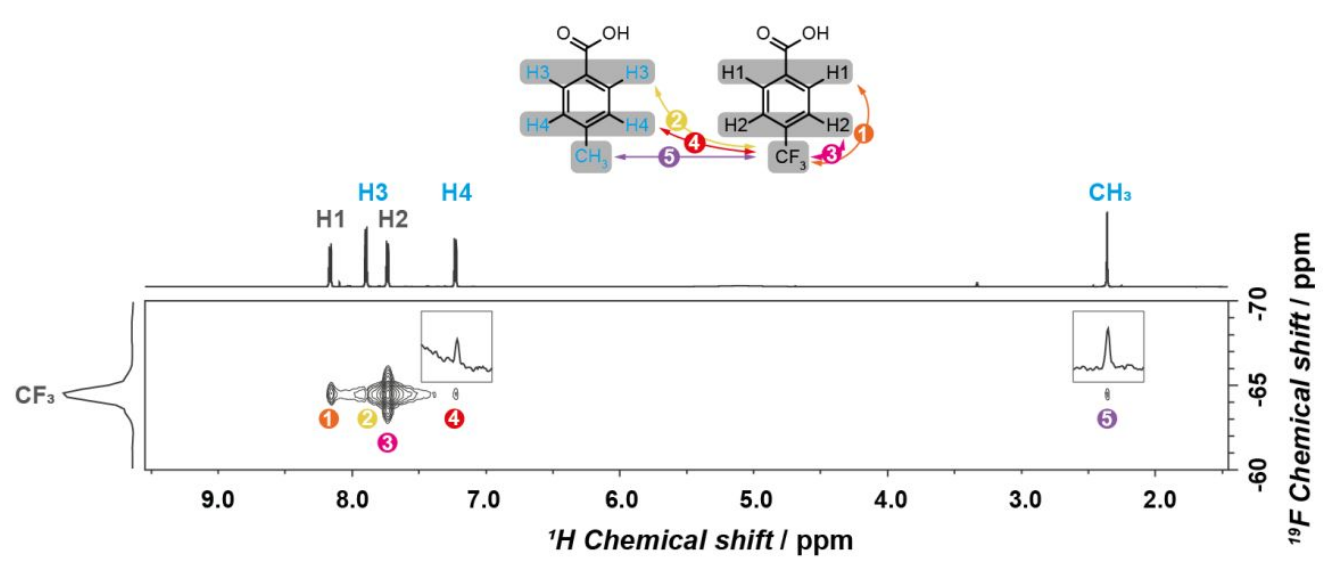

Fig. SI8. ${ }^{19} \mathrm{~F}-1 \mathrm{H}$ HOESY spectrum of saturated 4-methylbenzoic acid and 4-trifluoromethyl benzoic acid solution in methanol- $D_{4}$. Possible ${ }^{19} \mathrm{~F}^{-1} \mathrm{H}$ HOEs for this system are schematically indicated in the top section of the figure. Proton and fluorine atom labelling is given in the top section of the figure; protons of 4-methylbenzoic acid are indicated in red. The insets show inter-molecular HOE signal intensity. The inter-molecular HOE signal intensity ratio indicates that aggregates where methyl and trifluoromethyl groups are in close proximity are favoured.

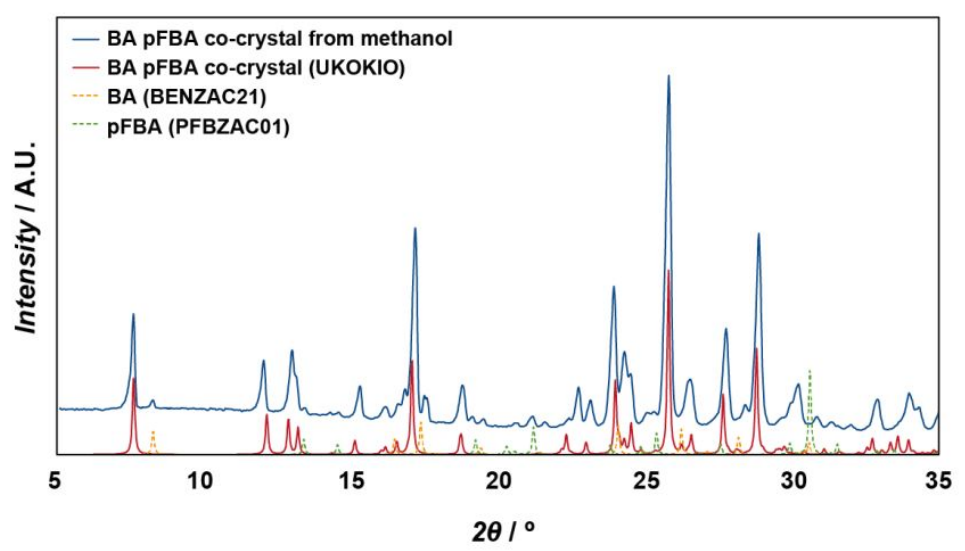

Fig. SI9. Experimental and theoretical powder X-ray diffraction patterns of BA pFBA co-crystal. Theoretical X-ray diffraction patterns of BA and $p F B A$ are shown as dashed lines. Minor fraction of $B A$ was identified in the co-crystal sample that was obtained after the NMR sample evaporative crystallisation. 


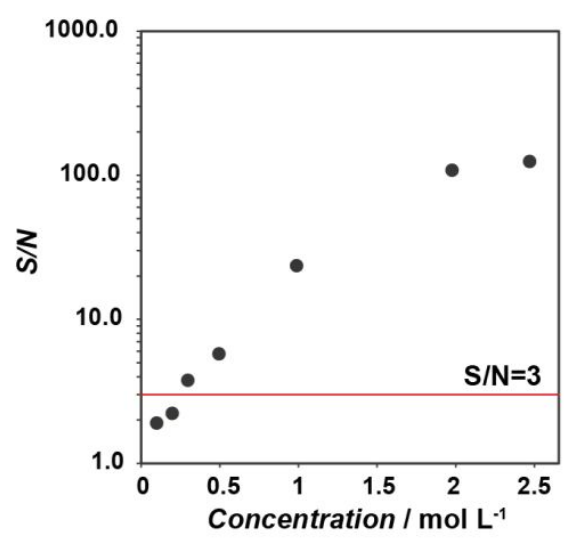

Fig. SI10. Signal to noise ratio for H2,H4:F2,F4 HOE signal as a function of 1:1 BA:pFBA methanol$\mathrm{D}_{4}$ solution concentration. Red line represents the signal to noise ratio 3. 